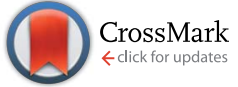

Cite this: RSC Adv., 2016, 6, 93715

Received 26th August 2016

Accepted 22nd September 2016

DOI: 10.1039/c6ra21476b

www.rsc.org/advances

\title{
Fractional molecular charge studied via molecular vibrational properties. Specific aspects in Jahn- Teller active molecular species $\uparrow$
}

\begin{abstract}
Ioan Bâldea $\ddagger^{*}$
In this paper, we report results obtained within the density functional theory (DFT) demonstrating that information on the fractional (non-integral) charge of a single molecule can be extracted from its vibrational properties. This is important because, unlike the former, the latter can be measured experimentally, e.g., via surface enhanced Raman spectroscopy (SERS). As a concrete example, we choose a benzene molecule and consider changes brought about by continuously varying the fractional charge from $q=0$ to $q=1$ corresponding to neutral and radical cation species. Molecular electronics or electrochemical platforms may offer experimental realizations of continuously tuning non-integral molecular charges. We found that changes in the vibrational frequencies $\omega_{v}(q)$ can be substantially larger than those recently measured by varying applied bias in molecular junctions. Our results indicate important qualitative and quantitative differences between the dependence $\omega_{v}=\omega_{v}(q)$ exhibited by vibrational modes that are JT active and those that are JT inactive; typically, the former is stronger and nonlinear, while the latter is weaker and linear.
\end{abstract}

\section{Introduction}

Understanding the interplay between electronic and vibrational degrees of freedom represents a key issue of chemical/ molecular physics. Interactions between charge carriers and molecular vibration degrees of freedom are important to understand electron transfer at interfaces and electron transport through molecules embedded in molecular junctions. ${ }^{1-7}$ By monitoring changes in vibrational properties brought about by the oxidation or reduction of a given neutral molecular species, valuable insight on the impact of local electronic density on various vibrational modes can be gained. However interesting, investigations done on molecular charge species existing in the natural form or easily prepared via ionization or electron attachment inherently suffer from an important limitation: only molecular species having an integral charge can be targeted. Thanks to recent advances in nano-/molecular electronics ${ }^{8-13}$ and electrochemistry, ${ }^{14,15}$ this limitation could be overcome. An excess charge transferred to fullerene molecules embedded in electromigrated molecular junctions of up to one tenth of an electron under an applied bias has been claimed from

Theoretische Chemie, Universität Heidelberg, Im Neuenheimer Feld 229, D-69120 Heidelberg, Germany

$\dagger$ Electronic supplementary information (ESI) available. See DOI: 10.1039/c6ra21476b

\$ Also at National Institute for Lasers, Plasmas, and Radiation Physics, Institute of Space Sciences, RO 077125, Bucharest-Măgurele, Romania, E-mail: ioan.baldea@ pci.uni-heidelberg.de concurrent transport-SERS (surface enhanced Raman spectroscopy) measurements. ${ }^{16}$ The fraction of the electron charge transfer has been estimated from changes in the vibrational properties. The latter can be measured via SERS or SEIRAS (surface enhanced infrared absorption spectroscopy) in nanojunctions consisting of one or a few molecules, because sharp electrodes act as plasmonic antennas and enormously enhance the local electric field. ${ }^{17}$ Furthermore, our recent theoretical calculations, ${ }^{18}$ which employed parameters extracted from experiments on EC-STM junctions based on viologen, ${ }^{9}$ indicated the possibility that an entire electron can be transferred. By continuously varying the overpotential-which is the counterpart of the gate potential of conventional electronics-, the LUMO occupancy of a molecule embedded in an EC-STM three terminal device based on an STM (scanning tunneling microscope) junction under EC (electrochemical control) can be smoothly tuned between zero for a neutral species and unity for a completely reduced species. ${ }^{18}$

The largest changes in vibrational frequencies deduced in ref. 16 are of the order of $\sim 10 \mathrm{~cm}^{-1}$. These changes are comparable to those measured in much older SERS studies ${ }^{19}$ on pyzarine molecules adsorbed on a macroscopic metallic silver electrode whose potential was varied in a certain range, wherein the main focus was on determining the orientation of the molecules on the metallic surface. Further theoretical work ${ }^{20}$ elaborated the effect of the electrode potential in terms of an effective molecular charge.

Substantially larger changes in molecular vibrational properties upon charge transfer can be expected in cases of 
molecules where the geometry of the charged species is qualitatively different from that of the neutral molecules. A benzene molecule is best suited to this purpose. Removing an electron from the ground state of the neutral $D_{6 \mathrm{~h}}$-symmetric benzene molecule yields a state that is twofold degenerate $\left(\tilde{\mathrm{X}}^{2} \mathrm{E}_{1 \mathrm{~g}}{ }^{21}\right)$ at the $D_{6 \mathrm{~h}}$ geometry. According to the Jahn-Teller (JT) theorem, ${ }^{22,23}$ this is a degenerate state of a nonlinear molecule, and its degeneracy is lifted by a JT distortion yielding a lowering of the symmetry; $D_{6 \mathrm{~h}} \rightarrow D_{2 \mathrm{~h}}$ in the case of benzene. ${ }^{24,25}$ Over decades, the Jahn-Teller distortion of a benzene radical cation has been the subject of detailed investigations. ${ }^{21,24,26-30}$

In the present work, we present results for a benzene molecule demonstrating that changes in vibrational frequencies driven by varying the fractional molecular charge of benzene can indeed be substantially larger that those observed so far in experiments. ${ }^{16}$ Furthermore, these results demonstrate that JTactive vibrational modes are qualitatively differently affected by charge removal compared to the JT-inactive ones. The latter are found to behave similarly to those reported recently ${ }^{31}$ for 1,4benzenedithiol, a molecule that does not possess JT-active vibrational modes. From a molecular electronics perspective, considering below positively charged species-fractional charges $0<q<+1$ ranging from the neutral $(q=0)$ to the radical cation $(q=+1)$ species - is also motivated: charge transport through benzene-based molecular junctions is often of the $\mathrm{p}$ type, i.e., mediated by the highest occupied molecular orbital (HOMO). ${ }^{5,32-34}$

\section{Computational details}

Most calculations done in this study have been performed running the SIESTA ${ }^{35}$ trunk-462 package $^{36}$ in parallel. ${ }^{37}$ Unlike most quantum chemical packages available, such as the GAUSSIAN 09 package $^{38}$ also partially employed in this study, SIESTA allows us to compute molecular vibrational properties for non-integer charge states. For calculations of the fractional charge states SIESTA uses a DFT (density functional theory) implementation developed in ref. 39. Similar to ref. 31, in our SIESTA-based calculations, we used the generalized gradient exchange correlation functional of Perdew, Burke, and Ernzerhof (GGA-PBE) ${ }^{\mathbf{4 0}}$ and split-valence TZP (split triple zeta polarized) basis sets for all atoms. Below we presented numerical results obtained using cubic $30 \times 30 \times 30 \AA$ cells and three dimensional real-space grids characterized by an energy cutoff of $800 \mathrm{Ry}$. The latter defines the maximum kinetic energy of the electronic plane wave basis set utilized; following SIESTA's manual, ${ }^{36}$ it will be referred to as the mesh cutoff below.

Employing cubic cells for a planar molecule like benzene is less common. It is motivated by the specific character of calculations to a charged system treated within an infinite periodic scheme utilized in the present paper, whose total energy converges very slowly with respect to the cell size. For cubic cells and only for these, a Madelung correction can be/is applied to the total energy, and this substantially improves convergence.

To obtain vibrational properties, SIESTA computes the dynamical matrix with a finite difference calculation of the forces $^{\mathbf{4 1}}$ via the VIBRA utility, which is part of the SIESTA package distribution. ${ }^{36}$ This method represents an alternative to the more popular method of obtaining phonons based on linear response DFT schemes. ${ }^{42}$

Table S1 of the ESI $\dagger$ comparatively presents the benzene vibrational frequencies computed via the presently adopted SIESTA/PBE/TZP method and other DFT as well as $a b$ initio (CCSD, MP2) methods. Albeit not dramatic, certain differences between the SIESTA values and those of the other methodsincluding those obtained employing GAUSSIAN 09 with keywords PBEPBE/TZVP-exist. They trace back to SIESTA's specific manner of numerically constructing localized orbitals; SIESTA's TZVP orbitals and GAUSSIAN's TZVP orbitals are not the same. However, we note that neither these differences nor the specific theoretical method producing the closest frequency values to experimental values represent the main issue in the present paper. What we want to emphasize is that, upon a nearly complete electron transfer, as it turned out to be possible by using three-terminal setups based on electrolyte gating $^{18}$ or asymmetric two terminal setups, ${ }^{43}$ bias-driven changes in vibrational frequencies can be substantially larger than what has been experimentally observed so far ${ }^{\mathbf{1 6}}$ and, hence, are easier to be measured by means of SERS or SEIRAS.

\section{Results and discussion}

\subsection{Preliminary remarks}

The calculations done for all fractional molecular charges $(0 \leq q$ $\leq 1$ ) investigated in the present paper refer to the lowest (ground) electronic state. A series of precautions has been taken in order to diminish as much as possible spurious numerical effects on the calculated vibrational properties, which-as delineated below and also encountered recently ${ }^{31}$-is a challenging task for SIESTA.

We relaxed the molecular geometries until the forces on all atoms were less than $0.1 \mathrm{meV} \AA^{-1}$. This is a much more severe condition than typical default force tolerances $\left(0.04 \mathrm{eV} \AA^{-1}\right.$ in SIESTA and $\sim 0.023 \mathrm{eV} \AA^{-1}$ in GAUSSIAN 09). The latter are often sufficient for relaxation studies, but the calculations of vibrational properties are usually much more demanding than those for relaxation.

Attention was paid to reduce egg-box effects. It might be helpful to remember that, to calculate various integrals, SIESTA uses a finite three-dimensional spatial grid. Because the relevant quantities (e.g., density or potential) have plane wave components beyond the finite mesh cutoff utilized, the translational invariance is broken (egg-box effect). This numerical effect disappears for infinitely fine grids (infinite mesh cutoff). For mesh cutoff values of $\sim 200-300 \mathrm{eV}$, egg-box effects on the total energy or relaxed geometry are normally completely insignificant; see Fig. 1 and discussion below.

However, eliminating such effects on the vibrational properties is much more difficult. We checked that changes to the cutoff values in the range of $300-1000 \mathrm{eV}$ do not conclusively reduce the degree of numerical noise exhibited by the vibrational properties. The results presented below have been obtained using a mesh cutoff of $800 \mathrm{eV}$. 


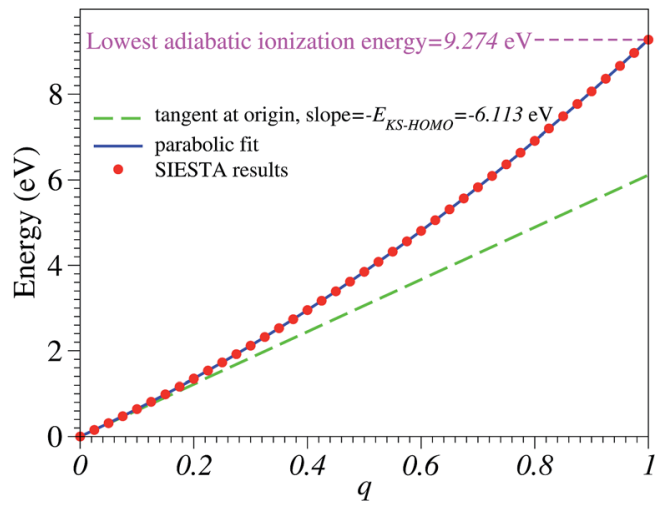

Fig. 1 The total energy versus the charge deficit $q$ computed with SIESTA is a smooth curve and yields a reasonable value for the lowest adiabatic ionization energy of the benzene molecule given in the legend. The total energy of the neutral molecule has been chosen as energy zero. See the main text for details.

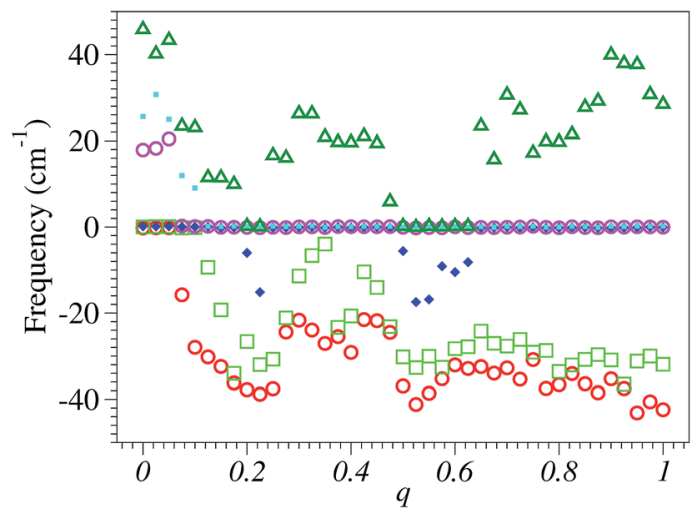

Fig. 2 The six lowest frequencies computed with SIESTA vs. electron charge deficit $q$. As they correspond to the three rigid translations and rotations of the entire molecule, they should be zero. The fact that they do not strictly vanish or are even imaginary (case represented here by negative values) is related to SIESTA's inherent numerical inaccuracies in dealing with phonons.

The effect of the finite difference procedure for force computations has also been considered. We determined that there is no substantial improvement by varying the displacement used for the computation of the force constant matrix (SIESTA keyword MD.FCDispl) from $0.01 \AA$ to $0.02 \AA$. For the results presented in this paper we used the value $0.01 \AA$.

Neither the precautions delineated above nor playing around with pseudopotentials - that is, using others than those actually utilized, obtained from translating the ABINIT pseudo's database available to download ${ }^{36}$-allowed us to eliminate some numerical inaccuracies/noise, which appear to be hardly avoidable in SIESTA calculations for vibrational properties while absent in considering more common electronic properties. The results depicted in Fig. 1 and 2, which were obtained by employing the same SIESTA settings/parameters, illustrate this contrast.

Numerical noise does not affect the total energy $\varepsilon(q)$ computed for various fractional charge states. In Fig. 1 we

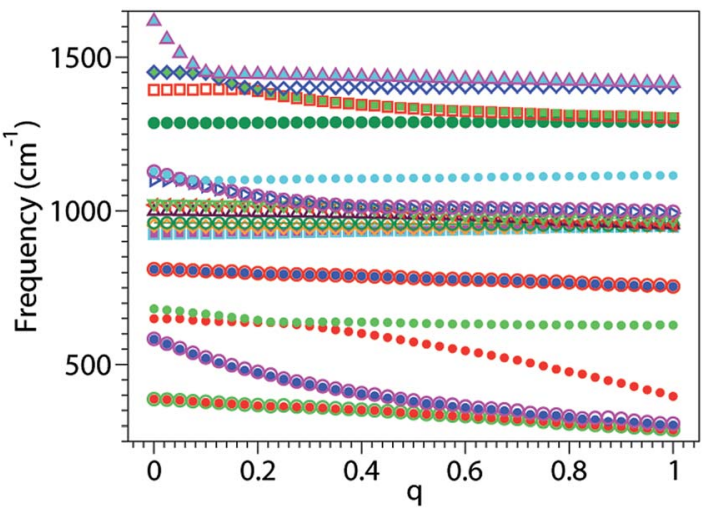

Fig. 3 The frequencies of the 24 relevant vibrational modes of the benzene molecule as a function of oxidation degree $q$. The points shown here have been obtained via SIESTA calculations as described in the main text. Not shown are the six high frequency modes $(\omega \sim 3000$ $\mathrm{cm}^{-1}$ ) related to $\mathrm{CH}$ stretching.

present the results for the total energy $\varepsilon(q)$ as a function of the oxidation degree $q$. The corresponding curve is a noise-free smooth curve, whose slope at the origin can be unambiguously extracted and yields a HOMO energy ${ }^{44-46}$

$$
E_{\mathrm{HOMO}}=\left.\frac{\partial \mathscr{E}(q)}{\partial q}\right|_{q \rightarrow 0^{+}}=-6.113 \mathrm{eV} .
$$

This value excellently agrees with the Kohn-Sham-(KS-) HOMO energy $\left.E_{\mathrm{HOMO}}\right|_{q=0}=-6.153 \mathrm{eV}$ computed for the neutral molecule $(q=0)$, as extracted from the SIESTA *.EIG file. As is amply documented ${ }^{47}$ although conceptually less problematic qualitatively than other KS "orbitals", the quantitative description of the physical HOMO by the KS-HOMO is rather poor; the above value(s) differs by more than $3 \mathrm{eV}$ from the value corresponding to the lowest adiabatic ionization energy obtained via $\Delta$-DFT calculations ${ }^{48-50} \mathrm{IP}=\mathscr{E}(q=1)-\mathscr{E}(q=0) \equiv \mathscr{E}_{\text {cation }}^{\text {equilibrium }}$ $\mathscr{E}_{\text {neutral }}^{\text {equilibrium }}=9.274 \mathrm{eV}$. This value of adiabatic ionization energy excellently agrees with the experimental value of the lowest adiabatic one-electron binding energy $(9.243 \mathrm{eV})$ measured by photoelectron spectroscopy. ${ }^{51}$ This agreement may not be taken too seriously, given the fact that more elaborate quantum chemical methods-like equation-of motion coupled cluster singles and doubles (EOM-CCSD), ${ }^{21,52}$ third-order algebraic diagrammatic construction $(\operatorname{ADC}(3)),{ }^{53}$ and outer valence Green's functions (OVGF) $)^{51,52}$ - do not yield such a "perfect" agreement; still, this fact demonstrates that SIESTA results for charged molecules may be reasonably trusted.

On the other side, in Fig. 2 we present the six lowest SIESTA frequencies, which correspond to the three rigid translations and rotations of the entire molecule; these frequencies should be strictly zero but they are not; a numerical inaccuracy that one cannot easily avoid. In fact, the problem with these values of six "should-be-zero" frequencies is also encountered in other quantum chemical packages. For instance, these are designated as "low frequencies" in the output files of GAUSSIAN 09, and their negative values mean imaginary frequencies. 

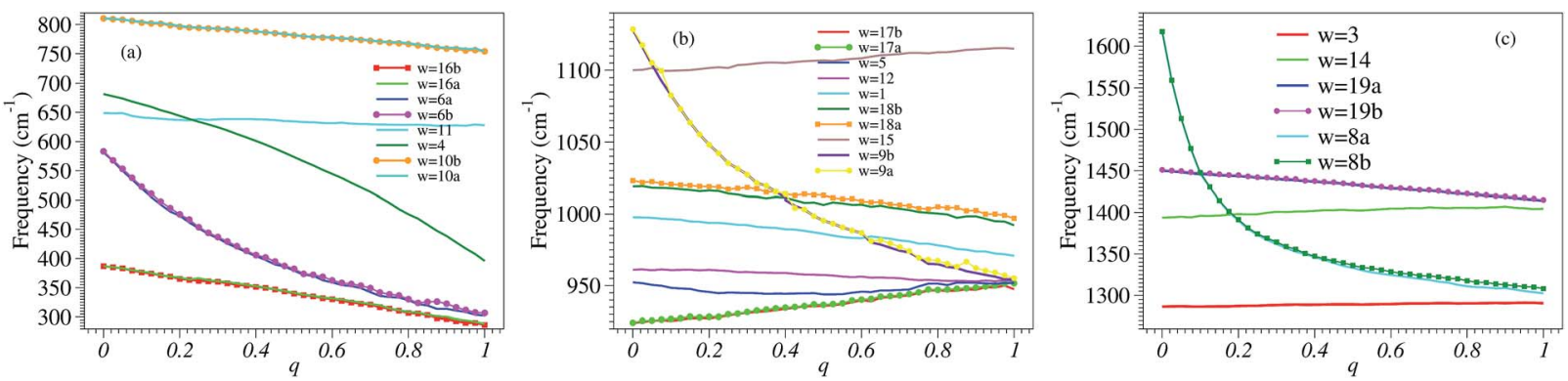

Fig. 4 The three panels $\mathrm{a}-\mathrm{c}$ of this figure were obtained by zooming in and splitting Fig. 3 in ranges of frequencies increasing from panel a to panel $c$, and joining the calculated points to build curves corresponding to well defined vibrational modes.

Due to the $D_{6 \mathrm{~h}}$ symmetry of the neutral benzene molecule, certain vibrational modes are twofold degenerate. As a result of the JT distortion, the benzene radical cation has a symmetry lower than the $D_{6 \mathrm{~h}}$-symmetry of the neutral benzene. Consequently, the twofold degeneracy of the vibrational modes of $E_{1, g}$ / $\mathrm{u}^{-}$and $\mathrm{E}_{2, \mathrm{~g} / \mathrm{u}}$-symmetries of the neutral species is lifted upon electron removal. Because the corresponding energy splittings are small, they cannot be distinguished at the noise level of SIESTA calculations. ${ }^{54}$ The mode symmetry indicated throughout this paper refers to the neutral benzene molecule.

However, in spite of the SIESTA limitations for phonon studies, the numerical noise more or less visible in all of the figures presented below does not prevent the formulation of clear conclusions on the relationship between the fractional electron charge and vibrational frequencies.

\subsection{Impact of charge removal on vibrational frequencies}

Our numerical results on the changes in the 24 relevant vibrational frequencies of the benzene molecule as a function of the electron deficit $q$ are collected in Fig. 3. For convenience-since otherwise the drawing scale needed in Fig. 3 would be detrimental for visibility-, the six highest frequency modes corresponding to $\mathrm{C}-\mathrm{H}$ stretching which are of lesser importance in the present context are not included in Fig. 3; their values are indicated in the last six lines of Table S1. $\dagger$

In the neutral benzene molecule, the lowest frequency mode is twofold degenerate and is denoted by $16 \mathrm{a} / 16 \mathrm{~b}$ in Wilson numbering ${ }^{55,56}$ and by 20 in Herzberg numbering. ${ }^{.7}$ This mode has $\mathrm{E}_{2 \mathrm{u}}$-symmetry in the point group $D_{6 \mathrm{~h}}$ and corresponds to an out-of-plane ring deformation (Fig. S4 of the ESI $\dagger$ ). Its frequency decreases upon electron removal. The frequency reduction is nearly linear with the electron deficit $q$; at complete oxidation $(q=1)$ this reduction amounts to $\sim 100 \mathrm{~cm}^{-1}$ (Fig. 4 a and S1a of the ESI $\dagger$ ). The motion of the various atoms does not exhibit any spectacular change upon varying the oxidation degree $q$; compare the panels of Fig. S4 in the ESI. $\uparrow$ This fact may be related to the linear $q$-dependence of the frequency, suggesting that electron removal basically acts as a first order perturbation.

Whether the frequencies decrease or increase by electron removal is not directly related to the mode's symmetry. In Fig. $4 \mathrm{~b}$ and S1c of the ESI, $\uparrow$ we present results revealing that, in contrast with the above case, the frequencies of the vibrational modes denoted by $17 \mathrm{a} / 17 \mathrm{~b}$ in Wilson labeling or 19 in Herzberg
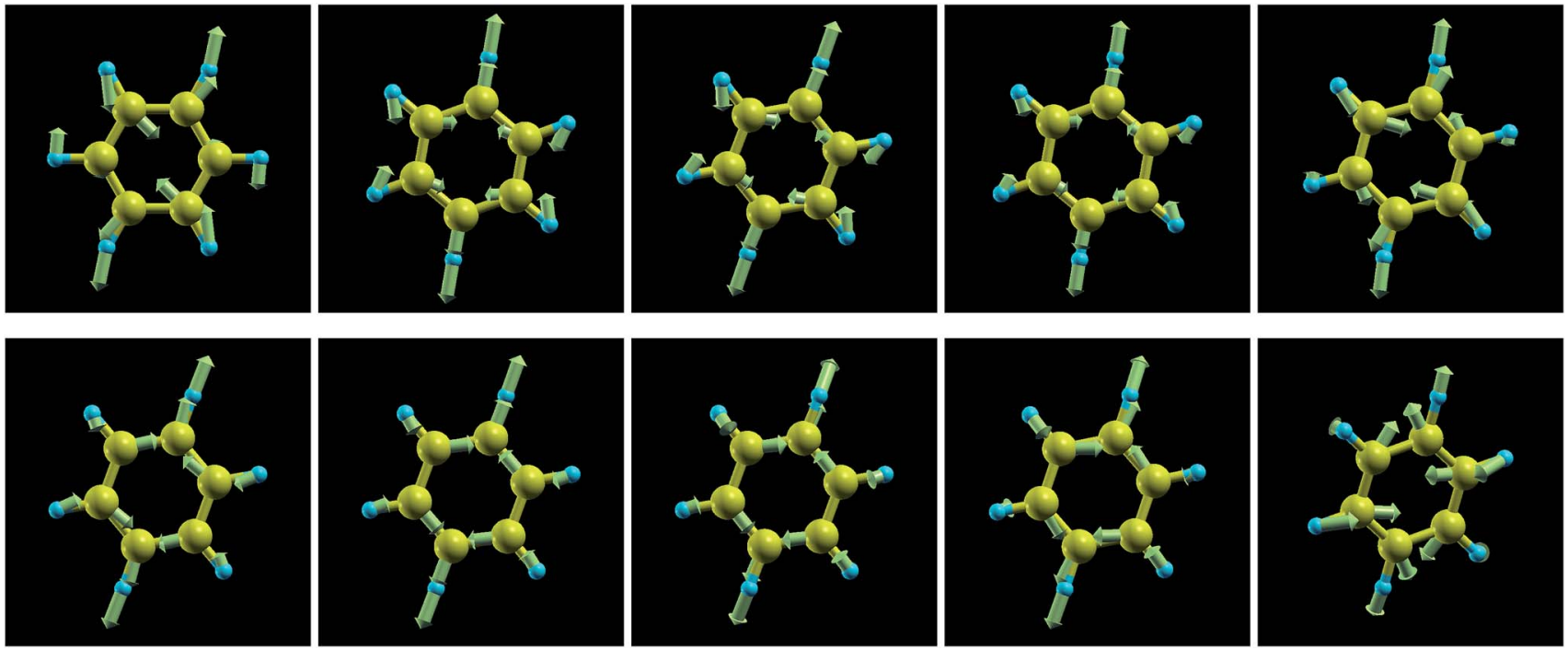

Fig. 5 Wilson mode 6a (Herzberg mode 18) for $q=0 ; 0.05 ; 0.1 ; 0.15 ; 0.3$ (upper row, from left to right) and $q=0.5 ; 0.7 ; 0.85 ; 0.9 ; 1.0$ (lower row, from left to right). For $q=0.9$ and $q=1.0$ SIESTA finds vibrations that are not strictly in-plane modes. 

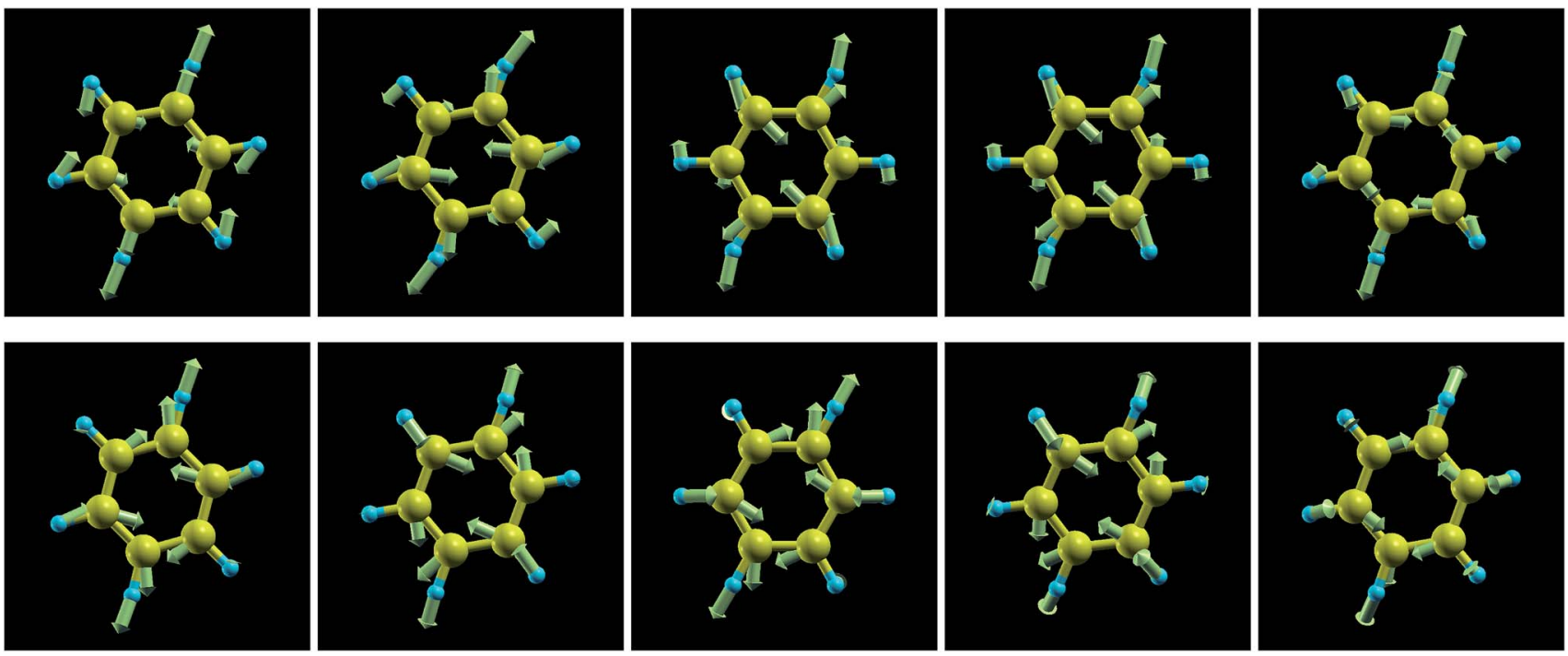

Fig. 6 Wilson mode $6 b$ (Herzberg mode 18) for $q=0 ; 0.05 ; 0.1 ; 0.15 ; 0.3$ (upper row, from left to right) and $q=0.5 ; 0.7 ; 0.85 ; 0.9 ; 1.0$ (lower row, from left to right). SIESTA predicts vibrations that are not strictly in plane for larger $q$ 's.

labeling, which also have $\mathrm{E}_{2 \mathrm{u}}$-symmetry in the neutral benzene, linearly increase by electron removal. These modes correspond to out-of-plane $\mathrm{CH}$ bendings. The frequency increases amount to $\sim 30 \mathrm{~cm}^{-1}$ upon complete oxidation.

Wilson modes $16 \mathrm{a} / 16 \mathrm{~b}$ and $17 \mathrm{a} / 17 \mathrm{~b}$ are neither infrared (IR) nor Raman active but are of interest because they induce interstate (pseudo-JT) vibronic couplings between a degenerate and a nondegenerate electronic state, e.g., between the $\tilde{\mathrm{B}}^{2} \mathrm{E}_{2 \mathrm{~g}}$ and $\tilde{\mathrm{C}}^{2} \mathrm{~A}_{2 \mathrm{u}}$ or between the $\tilde{\mathrm{D}}^{2} \mathrm{E}_{1 \mathrm{u}}$ and $\tilde{\mathrm{E}}^{2} \mathrm{~B}_{2 \mathrm{u}}$ electronic excited states of the radical cation. ${ }^{27,29}$ They are important in understanding the overlapping $\tilde{\mathrm{B}} / \tilde{\mathrm{C}}$ bands of the photoelectron spectrum and internal conversion dynamics..$^{27,29}$

The frequencies of the modes $6 \mathrm{a} / 6 \mathrm{~b}$ in Wilson numbering or 18 in Herzberg numbering, known to produce a strong JT coupling within the electronic lowest energy manifold $\tilde{\mathrm{X}}^{2} \mathrm{E}_{1 \mathrm{~g}}$, are more strongly affected by oxidation than the modes discussed above; see Fig. 4a and S2a of the ESI. $\dagger$ The various panels of Fig. 5 and 6 depict these modes for various values of oxidation degree $0 \leq q \leq 1$. In contrast to Fig. S4 of the ESI, $\dagger$ the fact that the motion amplitudes and directions of the various atoms change with $q$ is visible for these modes in Fig. 5 and 6. Wilson modes $6 \mathrm{a} / 6 \mathrm{~b}$ are Raman active. In neutral benzene they correspond to in-plane ring deformations ( $c f$. Fig. 5 and 6); at higher $q$-values, SIESTA found that these vibrations exhibit certain deviations from planarity.

The frequency that is most strongly affected by electron removal is that of Wilson mode $8 \mathrm{a} / 8 \mathrm{~b}$, which is mode 16 in Herzberg numbering (Fig. S5 and S6 of the ESI †); complete oxidation yields a frequency decrease amounting to $\sim 315 \mathrm{~cm}^{-1}$ (Fig. 4c and S2c of the ESI $\dagger$ ). This mode is Raman and JT active. As is visible in Fig. $4 c$, the curves for Wilson modes $8 \mathrm{a} / 8 \mathrm{~b}$ of $\mathrm{E}_{2 \mathrm{~g}^{-}}$ symmetry cross the curves for Wilson modes 19a/19b (mode 12 in Herzberg labeling; $\mathrm{E}_{1 \mathrm{u}}$-symmetry) and Wilson mode 14 (Herzberg mode 10; $\mathrm{B}_{2 \mathrm{u}}$-symmetry). The crossing is possible due to the different symmetries of these modes.
From this standpoint, Wilson modes 9a/9b (or 17 in Herzberg notation) are even more interesting. Although the change in frequency upon complete oxidation $\left(\sim 170 \mathrm{~cm}^{-1}\right)$ is smaller than that for Wilson mode $8 \mathrm{a} / 8 \mathrm{~b}$ (Herzberg mode 16 ), the curves for these modes (again $\mathrm{E}_{2 \mathrm{~g}}$ symmetry) exhibit three crossings ( $c f$. Fig. $4 \mathrm{~b}$ and S2b of the ESI $\dagger$ ) with those of Wilson mode 15 (9 in Herzberg notation; $\mathrm{B}_{2 \mathrm{u}}$ symmetry), Wilson modes $18 \mathrm{a} / 18 \mathrm{~b}$ (13 in Herzberg notation; $E_{1 u}$ symmetry), and the totally symmetric Wilson mode 1 ( 2 in Herzberg notation; $A_{1 g}$ symmetry).

All the crossings discussed above involve at least a twofold degenerate mode. The last example presented in this section demonstrates that this is not necessarily the case. Here we show two nondegenerate modes: Wilson mode 11 (Herzberg mode 4; $\mathrm{A}_{1 \mathrm{u}}$ symmetry) and Wilson mode 4 (Herzberg mode $8 ; \mathrm{B}_{1 \mathrm{~g}}$ symmetry).

In neutral benzene, the frequency of Wilson mode 11 (mode 4 in Herzberg notation) is somewhat higher then that of Wilson mode 4 (mode 8 in Herzberg notation). The former is substantially decreased by oxidation while the latter is nearly constant (Fig. 4a). Having different symmetries $\left(A_{1 u}\right.$ and $B_{2 g}$, respectively), they do not mix; the corresponding curves can and do cross at $q \geq 0.2$. However, because SIESTA does not use any symmetry in the calculations, a spurious mode mixing occurs at the crossing point; this is visible by comparing the panels corresponding for these two modes at $q=0.25$ (i.e., close to the crossing point, $c f$. Fig. 4 a) with the other panels of Fig. S7 and S8 of the ESI. $\dagger$

\section{Conclusion}

In this paper, we have studied theoretically the impact of removing an amount $0<q<1$ of electronic charge on the vibrational frequencies of a benzene molecule. We have determined that the changes in the phonon frequencies are 
substantially larger than found in recent experiments ${ }^{\mathbf{1 6}}$ in nanojunctions based on fullerene.

As an important result to be noted, we have demonstrated that vibrational frequencies $\omega_{\mathrm{v}}=\omega_{\mathrm{v}}(q)$ are affected in a different manner by the charge deficit $q$, depending on whether the vibrational modes are Jahn-Teller active or not.

Basically, for JT-inactive modes, the dependence $\omega_{\mathrm{v}} v s . q$ is linear. This result gives microscopic support to our recent conjecture, ${ }^{18}$ amounting to considering the vibrational frequencies in a fractional charge state as a linear interpolation between the frequencies of the neutral and (in the concrete case investigated here) radical cation species with a weight determined by the charge deficit $q$. Rephrasing, the charge removal acts on the vibrational frequencies as a first-order perturbation. So, the conjecture of ref. 18 turned out to provide a reasonable framework for interpreting experimental data in nanojunctions based on mostly utilized molecules wherein charge transfer does not yield too strong perturbations, like the presently considered JT distortion. In assessing its operational utility for quantitative purposes one should take into account that, in order to make the DFT-computed values comparable with the measured vibrational frequencies, empirical scaling factors have to be applied, ${ }^{58}$ and these scaling factors may depend on the charge species. ${ }^{59}$

For JT-active modes, the dependence $\omega_{\mathrm{v}} v s . q$ has been found to be stronger and substantially deviates from linearity. This nonlinearity found, a qualitatively different aspect, is in agreement with the overall view on JT distortion as a source of qualitative different behavior.

Many vibrational modes in the ground state of the benzene radical cation have been found to have frequencies substantially different from those of the neutral benzene in the ground state. From the point of view of quantum dynamical simulations, this finding is also important. $A b$ initio quantum dynamical approaches to the benzene $e^{21,24,26-30}$ or halogen substituted benzene ${ }^{30,60,61}$ radical cation are often based on normal vibrational coordinates of the neutral species. In the language of those normal coordinates, the present result implies substantial quadratic couplings in the lowest energy manifold $\tilde{\mathrm{X}} \mathrm{E}_{1 \mathrm{~g}}$ of the radical cation. From this perspective, augmenting quantum dynamical simulations done for the benzene radical cation ${ }^{\mathbf{2 1 , 2 4 , 2 6 - 3 0}}$ by also including quadratic couplings, as done for halogen substituted counterparts, ${ }^{30,60,61}$ can be a meaningful next step.

\section{Acknowledgements}

Financial support for this research provided by the Deutsche Forschungsgemeinschaft (grant BA 1799/3-1) is gratefully acknowledged. This work was partially performed on the computational resources bwUniCluster and bwForCluster/ JUSTUS-HPC facility funded by the Ministry of Science, Research and Arts Baden-Württemberg and the Universities of the State of Baden-Württemberg, Germany, within the framework program bwHPC. ${ }^{37}$

\section{Notes and references}

1 A. Nitzan, Annu. Rev. Phys. Chem., 2001, 52, 681-750.
2 J. Jiang, M. Kula, W. Lu and Y. Luo, Nano Lett., 2005, 5, 15511555.

3 M. Galperin, M. A. Ratner, A. Nitzan and A. Troisi, Science, 2008, 319, 1056-1060.

4 V. Coropceanu, R. S. Sánchez-Carrera, P. Paramonov, G. M. Day and J.-L. Brédas, J. Phys. Chem. C, 2009, 113, 4679-4686.

5 H. Song, M. A. Reed and T. Lee, Adv. Mater., 2011, 23, 15831608.

6 R. Härtle and M. Thoss, in Molecular Electronics: An Experimental and Theoretical Approach, ed. I. Bâldea, Pan Stanford, 2015, ch. 5, pp. 155-221.

7 D. Xiang, X. Wang, C. Jia, T. Lee and X. Guo, Chem. Rev., 2016, 116, 4318-4440.

8 A. Alessandrini, S. Corni and P. Facci, Phys. Chem. Chem. Phys., 2006, 8, 4383-4397.

9 I. V. Pobelov, Z. Li and T. Wandlowski, J. Am. Chem. Soc., 2008, 130, 16045-16054.

10 S. Wang, M. Ha, M. Manno, C. D. Frisbie and C. Leighton, Nat. Commun., 2012, 3, 1210.

11 W. Xie, S. Wang, X. Zhang, C. Leighton and C. D. Frisbie, Phys. Rev. Lett., 2014, 113, 246602.

12 A. Alessandrini and P. Facci, in Molecular Electronics: An Experimental and Theoretical Approach, ed. I. Bâldea, Pan Stanford, 2015, ch. 9, pp. 325-352.

13 J. M. Artés-Vivancos, J. Hihath and I. Díez-Pérez, in Molecular Electronics: An Experimental and Theoretical Approach, ed. I. Bâldea, Pan Stanford, 2015, ch. 8, pp. 281-324.

14 A. Alessandrini and P. Facci, Eur. Polym. J., 2016, DOI: 10.1016/j.eurpolymj.2016.03.028.

15 J. Izquierdo and C. Kranz, Eur. Polym. J., 2016, DOI: 10.1016/ j.eurpolymj.2016.05.016.

16 Y. Li, P. Doak, L. Kronik, J. B. Neaton and D. Natelson, Proc. Natl. Acad. Sci. U. S. A., 2014, 111, 1282-1287.

17 P. Mühlschlegel, H.-J. Eisler, O. J. F. Martin, B. Hecht and D. W. Pohl, Science, 2005, 308, 1607-1609.

18 I. Bâldea, Phys. Chem. Chem. Phys., 2014, 16, $25942-25949$.

19 J. Soto, D. J. Fernández, S. P. Centeno, I. L. Tocón and J. C. Otero, Langmuir, 2002, 18, 3100-3104.

20 F. Avila, C. Ruano, I. López-Tocón, J. F. Arenas, J. Soto and J. C. Otero, Chem. Commun., 2011, 47, 4213-4215.

21 M. Döscher, H. Köppel and P. G. Szalay, J. Chem. Phys., 2002, 117, 2645-2656.

22 H. A. Jahn and E. Teller, Proc. R. Soc. London, Ser. A, 1937, 161, 220-235.

23 R. Englman, The Jahn-Teller effect in molecules and crystals, Wiley, New York, 1972.

24 H. Müller, H. Köppel and L. S. Cederbaum, New J. Chem., 1993, 17, 7-29.

25 K. Müller-Dethlefs and J. B. Peel, J. Chem. Phys., 1999, 111, 10550.

26 M. Döscher and H. Köppel, Chem. Phys., 1997, 225, 93-105.

27 H. Köppel, M. Döscher, I. Bâldea, H.-D. Meyer and P. G. Szalay, J. Chem. Phys., 2002, 117, 2657-2671.

28 H. Köppel, I. Bâldea and P. Szalay, in Advances in Quantum Chemistry, ed. J. R. Sabin and E. J. Braendas, Academic Press, 2003, vol. 44, pp. 199-217. 
29 I. Bâldea and H. Köppel, J. Chem. Phys., 2006, 124, 064101.

30 I. Bâldea, J. Franz and H. Köppel, J. Mol. Struct., 2007, 838, 94-99.

31 I. Bâldea, Chem. Phys., 2016, DOI: 10.1016/ j.chemphys.2016.08.024.

32 H. Song, Y. Kim, Y. H. Jang, H. Jeong, M. A. Reed and T. Lee, Nature, 2009, 462, 1039-1043.

33 Z. Xie, I. Bâldea, C. Smith, Y. Wu and C. D. Frisbie, ACS Nano, 2015, 9, 8022-8036.

34 M. Tsutsui, T. Morikawa, Y. He, A. Arima and M. Taniguchi, Sci. Rep., 2015, 5, 11519.

35 J. M. Soler, E. Artacho, J. D. Gale, A. Garcia, J. Junquera, P. Ordejon and D. Sanchez-Portal, J. Phys.: Condens. Matter, 2002, 14, 2745-2779.

36 Available at http:/www.icmab.es/dmmis/leem/siesta/.

37 bwHPC and bwHPC-C5, http://www.bwhpc-c5.de/, Computational resources funded by the Ministry of Science, Research and Arts Baden-Württemberg and the Universities of the State of Baden-Württemberg, Germany, and the German Research Foundation (DFG).

38 M. J. Frisch, G. W. Trucks, H. B. Schlegel, G. E. Scuseria, M. A. Robb, J. R. Cheeseman, G. Scalmani, V. Barone, B. Mennucci, G. A. Petersson, H. Nakatsuji, M. Caricato, X. Li, H. P. Hratchian, A. F. Izmaylov, J. Bloino, G. Zheng, J. L. Sonnenberg, M. Hada, M. Ehara, K. Toyota, R. Fukuda, J. Hasegawa, M. Ishida, T. Nakajima, Y. Honda, O. Kitao, H. Nakai, T. Vreven, J. A. Montgomery Jr, J. E. Peralta, F. Ogliaro, M. Bearpark, J. J. Heyd, E. Brothers, K. N. Kudin, V. N. Staroverov, T. Keith, R. Kobayashi, J. Normand, K. Raghavachari, A. Rendell, J. C. Burant, S. S. Iyengar, J. Tomasi, M. Cossi, N. Rega, J. M. Millam, M. Klene, J. E. Knox, J. B. Cross, V. Bakken, C. Adamo, J. Jaramillo, R. Gomperts, R. E. Stratmann, O. Yazyev, A. J. Austin, R. Cammi, C. Pomelli, J. W. Ochterski, R. L. Martin, K. Morokuma, V. G. Zakrzewski, G. A. Voth, P. Salvador, J. J. Dannenberg, S. Dapprich, A. D. Daniels, O. Farkas, J. B. Foresman, J. V. Ortiz, J. Cioslowski, and D. J. Fox, Gaussian 09, Revision B.01, Gaussian, Inc., Wallingford CT, 2010.

39 J. P. Perdew, R. G. Parr, M. Levy and J. L. Balduz, Phys. Rev. Lett., 1982, 49, 1691-1694.
40 J. P. Perdew, K. Burke and M. Ernzerhof, Phys. Rev. Lett., 1996, 77, 3865-3868.

41 G. J. Ackland, M. C. Warren and S. J. Clark, J. Phys.: Condens. Matter, 1997, 9, 7861-7872.

42 S. Baroni, P. Giannozzi and A. Testa, Phys. Rev. Lett., 1987, 58, 1861-1864.

43 I. Bâldea, Phys. Chem. Chem. Phys., 2015, 17, 15756-15763.

44 J. F. Janak, Phys. Rev. B: Solid State, 1978, 18, 7165-7168.

45 G. K.-L. Chan, J. Chem. Phys., 1999, 110, 4710-4723.

46 A. J. Cohen, P. Mori-Sánchez and W. Yang, Phys. Rev. B: Condens. Matter Mater. Phys., 2008, 77, 115123.

47 R. G. Parr and W. Yang, Density-Functional Theory of Atoms and Molecules, Oxford University Press, Clarendon, Oxford, 1989.

48 R. O. Jones and O. Gunnarsson, Rev. Mod. Phys., 1989, 61, 689-746.

49 I. Bâldea, Europhys. Lett., 2012, 99, 47002.

50 I. Bâldea, Faraday Discuss., 2014, 174, 37-56.

51 P. Baltzer, L. Karlsson, B. Wannberg, G. Öhrwall, D. Holland, M. MacDonald, M. Hayes and W. von Niessen, Chem. Phys., 1997, 224, 95-119.

52 I. Bâldea, Phys. Chem. Chem. Phys., 2015, 17, 20217-20230.

53 M. S. Deleuze, A. B. Trofimov and L. S. Cederbaum, J. Chem. Phys., 2001, 115, 5859-5882.

54 For reader's convenience, both Wilson ${ }^{55}$ and Herzberg ${ }^{57}$ labels of the various vibrational modes of benzene will be indicated.

55 E. B. Wilson, Phys. Rev., 1934, 45, 706-714.

56 G. Varsanyi, in Assignments for Vibrational Spectra of Seven Hundred Benzene Derivatives, ed. I. Land, Adam Hilger, London, 1974, vol. 1, p. 1.

57 G. Herzberg, Molecular spectra and molecular structure, Infrared and Raman spectra of polyatomic molecules, Van Nostrand, Reinhold, New York, 1945, vol. 2.

58 A. P. Scott and L. Radom, J. Phys. Chem., 1996, 100, 1650216513.

59 I. Bâldea, H. Köppel and W. Wenzel, Phys. Chem. Chem. Phys., 2013, 15, 1918-1928.

60 I. Bâldea, J. Franz, P. G. Szalay and H. Köppel, Chem. Phys., 2006, 329, 65-75.

61 E. Gindensperger, I. Bâldea, J. Franz and H. Köppel, Chem. Phys., 2007, 338, 207-219. 